\title{
INTERPRETACIÓN DE LOS CONOCIMIENTOS QUE INFLUYEN EN LA ADHERENCIA A LA DIETOTERAPIA EN ADULTOS MAYORES CON DIABETES TIPO 2 DE UNA COMUNA RURAL
}

\author{
INTERPRETATION OF THE KNOWLEDGE ABOUT TYPE 2 \\ DIABETES AND COMPLIANCE TO DIETOTHERAPY AMONG \\ ELDERLY LIVING IN RURAL AREAS SUFFERING FROM THIS DISEASE
}

\author{
Claudia Troncoso P. (1), Mauricio Sotomayor C. (1), Fernanda Ruiz H. (2), Carla Zúñiga T. (2) \\ (1) Carrera de Nutrición y Dietética. Facultad de Medicina. Universidad Católica de la Santísima Concepción. \\ (2) Estudiante de la Carrera de Nutrición y Dietética. Facultad de Medicina. \\ Universidad Católica de la Santísima Concepción
}

\begin{abstract}
Type 2 diabetes is one of the most prevalent chronic diseases in the country. Among the strategies for its treatment is education, a tool presented by the health team to modify risky behavior on these people, providing users information and some strategies for their self-care. The objective of this study was to analyze the different factors associated to the knowledge of the pathology that influences the compliance to diet therapy treatment among elderly suffering from type 2 diabetes, who are regularly checked by a nutritionist in Yumbelis Health Public System. Through a qualitative phenomenological-type study, a semi-structured interview was carried out to 14 people in standard conditions assessing the knowledge about the pathology in terms of the consequences and the treatment to follow. However, the knowledge does not determine whether people will choose to follow diet therapy, with the exception of people with knowledge of the consequences of the disease. In the final analysis, the knowledge of the pathology does not induce the majority of the elderly from rural areas to follow diet therapy.
\end{abstract}

Key words: type 2 diabetes, education, rural elderly, qualitative studies.

Este trabajo fue recibido el 1 de Septiembre de 2008 y aceptado para ser publicado el 10 de Noviembre de 2008

\section{INTRODUCCIÓN}

Las enfermedades crónicas no transmisibles (ECNT) son un problema de salud creciente a nivel mundial y nacional(1). Estas patologías constituyen una gran carga social y económica, y pueden generar diversos grados de incapacidad; puede presentar una etiología originada por distintas condicionantes(2). En el informe elaborado por la Organización Mundial de la Salud (OMS)(3) el año 2005, se determinó que las enfermedades crónicas son la principal causa de mortalidad en un gran número de países, (4) especialmente en aquellos de ingresos bajos y medios. El $60 \%$ de todas las defunciones se deben a algún tipo de enfermedad crónica, (3) como hipertensión arterial, diabetes tipo 2 (DM2), enfermedades coronarias, accidentes cerebrovasculares, obesidad o distintos tipos de cáncer (5). Este fenómeno se atribuye a varios cambios que han experimentado la mayor parte de los países, especialmente aquellos desarrollados o en vías de este desarrollo. Esta transición epidemiológica se ha producido por el control de la desnutrición infantil, las enfermedades infecciosas, baja natalidad, el aumento de las expectativas de vida y la adquisición progresiva de un modelo de vida poco saludable (1,5-6).

Latinoamérica (7) y en especial Chile, es parte de este cambio de perfil epidemiológico(1) Existe un aumento en la prevalencia de enfermedades crónicas no transmisibles en el adulto. (6); así, la Encuesta Nacional de Salud (ENS) realizada en el año 2003 refleja la actual situación en la población del país. Por lo anterior, el Ministerio de Salud definió entre sus metas sanitarias para 
los años 2000 al 2010 (8) el mejorar la calidad de vida de las personas, especialmente en aquellos que padezcan de alguna enfermedad crónica como es la DM tipo 2, formulando directrices tendientes a disminuir la magnitud de la discapacidad provocada por esta patología.

La Diabetes se caracteriza por deficiencias en la secreción y/o acción de la insulina(9). Se presenta como un desorden metabólico crónico, caracterizado por niveles persistentemente elevados de glucosa en la sangre(10). La hiperglicemia crónica de la diabetes se asocia a muerte prematura, daño en diversos órganos, especialmente ojos, corazón y vasos sanguíneos, entre otros(11). Actualmente, 35 millones de personas en Latinoamérica y el Caribe sufren de esta enfermedad. La Organización Panamericana de la Salud (OPS) estima que para el año 2025, esta cifra aumentará a 64 millones(12). La prevalencia según la Encuesta Nacional de Salud (ENS) del año 2003 en el país es de un 4.3\%. La tasa de mortalidad en el año 2004 fue de 21.4 x 100.000 habitantes (3415 personas fallecidas por esta causa), de las cuales 1606 corresponden a personas entre 65 a 79 años, según datos entregados en el año 2005 por el Departamento de Estadísticas e Información de Salud (DEIS)(13). Causa en gran porcentaje de discapacidad en la población (42\% visual y 19\% por amputaciones no traumáticas)(8).

La prevención y el tratamiento de esta patología se basa en la atención multidisciplinaria del equipo médico, a través de modificaciones en sus estilos de vida; incorporando o incrementando la realización de actividad física; modificaciones de la dieta alimentaria y en ocasiones el uso de fármacos. Una acción efectiva ante el problema de salud que representa esta enfermedad, exige un enfoque integral entre prevención, cuidado y educación (14-20). La atención y la educación se encuentran entre los aspectos más importantes en la lucha y tratamiento contra esta patología. Se ha demostrado que la educación en diabetes logra un efecto positivo en los pacientes, reduciendo las tasas de mortalidad y morbilidad innecesarias debidas a su control deficiente (14).

Para que exista adherencia al tratamiento en la DM2 se necesita de la participación y el compromiso activo de la persona con su salud. Sin embargo, esta adherencia en un número importante de usuarios no se presenta, ya sea por condicionantes en este último (familiares, sociales, económicos, entre otros factores) o por causas originadas por miembros del equipo de salud (carencia de tiempo, recursos económicos o conocimientos)(23). Una de las alternativas destinadas al aumento de la adherencia a dietas en la práctica clínica puede ser ofreciendo al usuario una amplia alternativa de regímenes con opciones, de manera que la persona pueda establecer sus propias preferencias y adaptar la dieta a su estilo de vida y riesgo cardiovascular (24). La alimentación programada más la actividad física, son la base del tratamiento no farmacológico de la diabetes, sin estos pilares, resulta difícil un completo control metabólico (25).

La presencia de DM2 en adultos mayores es favorecida por la disminución de la actividad física, el menor consumo de carbohidratos complejos y el aumento de alimentos de alta densidad energética, así como el incremento de la adiposidad con la reducción de la masa corporal magra (21).

En estos últimos años, Chile está inmerso en un cambio demográfico, aumentando en forma exponencial la población de adultos mayores. Cifras entregadas por el INE (Instituto Nacional de Estadísticas) señalan que la población de personas mayores en la región de Bío-Bío corresponde a un 11,4\%. La comuna de Yumbel, es una zona rural ubicada en la Provincia del Bío-Bío, Chile. Presenta una densidad poblacional de 20.498 habitantes, de los cuales 3.297 personas son adultos mayores, correspondiente a un $16,08 \%$ del total de la población, siendo ésta la comuna con el mayor porcentaje de adultos mayores en la provincia (22).

La importancia que presenta la adherencia al tratamiento no farmacológico en adultos mayores con DM2, generó este estudio que tuvo como objetivo interpretar los factores asociados a los conocimientos que influyen en la adherencia al tratamiento dietoterapéutico en usuarios adultos mayores, controlados por nutricionista en la Atención Primaria de Salud de la comuna de Yumbel, durante el primer semestre del año 2008.

\section{SUJETOS Y MÉTODO}

Tipo de estudio y población estudiada: Se realizó estudio cualitativo fenomenológico en adultos mayores con diagnóstico clínico de DM 2 pertenecientes a la comuna de Yumbel, zona rural de la Región del BíoBío, Chile.

Los criterios de selección para este estudio fueron presentar 65 años o más de vida; diagnóstico clínico de DM2; usuarios de establecimiento perteneciente a la Atención Primaria en Salud de la comuna de Yumbel; tratados farmacológicamente con hipoglicemiantes orales (HGO) y controlados por nutricionista, además de la firma del consentimiento informado.

El cálculo de la muestra se obtuvo mediante saturation point, que en esta investigación se determinó cuando el $50 \%$ de las respuestas se comenzaban a repetir. El punto de saturación se presentó con 14 entrevistas realizadas en condiciones estándar por los investigadores en usuarios que cumplían con los criterios de selección 
del estudio.

Método: El instrumento utilizado para obtener la información en estos adultos mayores correspondió a una entrevista semi-estructurada de 13 preguntas en las que se utilizó un lenguaje claro, sencillo y simple, acorde al grupo en estudio, aplicado en condiciones estándar, previa prueba piloto. Las entrevistas fueron realizadas en los respectivos domicilios de los usuarios durante el mes de marzo del año 2008. Las respuestas entregadas se grabaron para ser posteriormente traspasadas a formato papel. Luego fueron analizadas por los investigadores a través del análisis semántico estructural, el que consiste en la identificación de códigos de bases, los que se obtienen de los discursos obtenidos en cada entrevista.

\section{RESULTADOS}

Los resultados se obtienen del análisis del discurso realizado por las personas mayores entrevistadas y de los objetivos planteados para esta investigación.

\section{1.- Conocimiento acerca de la patología crónica que padecen}

La mayoría de los entrevistados demuestran poseer conocimientos básicos acerca de su patología de base, que se refleja a través de respuestas simples como: "... una enfermedad..."; “...es una enfermedad peligrosa...” y otorgando calificativos como "...es algo que es muy malo..."; “...es un daño muy grande...”; “... que es larga y no tiene cura...".

Además, mencionan características claves de la DM tipo 2 en respuestas como: “... que hecha a perder todo el sistema..."; “... lo encachado que no duele... no se siente mal uno con ella...". Estas respuestas se interpretan a que la enfermedad es percibida como invasiva para múltiples órganos y asintomática.

Otra interpretación acerca de los conocimientos de la patología que presentan es la cronicidad de ésta: “... que dura para toda la vida....”, “...que por mucho tiempo dura...”. Gran parte de los entrevistados, además, identifican la historia familiar como un factor de riesgo en el desarrollo de la enfermedad: “... mi hermana también tiene, mi hermana mayor, y eso yo le decía a mi mamá, la media herencia que nos va a dejar...”; “... aunque yo tenía claro que iba a tener diabetes porque yo vengo de una familia diabética, entonces yo sabía que iba a tener diabetes...".

\section{2.- Conocimiento acerca del tratamiento dietoterapéutico en la DM2}

Gran parte de los entrevistados asocian el concepto "dieta" a un tipo de restricción alimentaria: “... son muchas cosas..."; “...me voy a morir de hambre...”; “...una dieta es privarse de comida...”. Este concepto es interpretado por la mayoría de los usuarios entrevistados independiente de que presenten diabetes como patología de base.

En la concepción de "dieta" como parte del tratamiento en la DM2, gran parte de los entrevistados consideran que principalmente se debe restringir el consumo de carbohidratos de absorción rápida: “...que no coma azúcar...”; “...bueno, lo del azúcar, el pan, la fruta todo eso de poquito por que más mal le hacen a una...y...la mermelada tampoco..." y que la cantidad de alimentos debe ser reducida "...que si se va a servir un racimo de uva no... solo unos granitos..."; “. . .bueno me han dicho sobre todo en cuanto a la fruta que debo comer, pero una cantidad un poco pequeña...".

\section{3.- Conocimientos acerca de efectos nocivos provocados por la no adherencia al tratamiento dietoterapéutico de la patología de base}

Según la mayoría de las respuestas entregadas por los pacientes, existirían conocimientos respecto al tema en estudio. Los entrevistados demuestran saber que existen consecuencias o complicaciones de la enfermedad. Este aprendizaje es obtenido principalmente a través del conocimiento popular, es decir, en base a experiencias de otras personas: “... por que mi mamá se quedó ciega por eso y se murió de eso...”; “.... a una persona ya le han cortado un dedo...". En algunos casos el conocimiento acerca de los daños que podría producir la no adherencia a la dietoterapia, es adquirido a través de la información entregada por profesionales de la salud, especialmente durante el control nutricional: "...uno sabe por lo que le dicen ahí en el hospital ahí... la... la señorita...”. Además, existe conocimiento acerca de órganos que pueden ser afectados y generar dichas complicaciones: “...partiendo por los ojos que se dañan...también lo de los pies...”; “...me puedo dañar o tener problema con los riñones...".

\section{4.- Conocimientos acerca de los beneficios que otorga la adherencia al tratamiento dietoterapéutico en la DM2}

En el caso de esta investigación, gran parte de las respuestas entregadas por los entrevistados están dirigidas principalmente al control metabólico de la patología. Esta referencia se obtiene a partir de respuestas como: “...que me han controlado bien porque...porque antes me subía mucho la glicemia y ahora de que estoy casi con pura dieta, he sufrido como dos veces no más, pero he estado controlada..."; " ...yo me he sentido bien y he estado controlado, eso yo creo, que uno cuando come bien se mantiene la diabetes...". 


\section{DISCUSIÓN}

Los miembros del equipo de salud debemos considerar que los usuarios o sujetos en estudio, viven y perciben el mundo en forma muy distinta a la nuestra, por lo que se deben considerar sus creencias o las influencias que estas presentan en las distintas manifestaciones de la dicotomía salud-enfermedad(26). La metodología cualitativa permite conocer la percepción, que los sujetos tienen de sus vivencias, de una manera que los diseños cuantitativos no lo permiten (27). Este diseño facilita, además, la comprensión del sentido de las representaciones sociales en el lenguaje de los sujetos que conforman la sociedad y en base a las situaciones que estos se encuentran o vivencian (28-29).

Parte del tratamiento no farmacológico en usuarios con DM2 se basa en intervenciones educativas, las que a corto o mediano plazo, presentan efectos en el control de la patología, además de proporcionar conductas y conocimientos que permiten realizar acciones destinadas al autocontrol (30-32). Estudios realizados en base a intervenciones educativas tienen como resultado el aumento en la adquisición de conocimientos y la mejoría en el control metabólico de usuarios con DM2 (33-36). Sin embargo, gran parte de estas investigaciones no son realizadas en adultos mayores.

La información o conocimientos que presenta el grupo en estudio acerca de la patología es básico, identificando de manera general en que consiste la enfermedad. Sin embargo, los entrevistados demuestran conocer conceptos específicos de su patología, principalmente aquellos efectos negativos que ésta puede ocasionar en individuos no adheridos a los tratamientos, tanto dietoterapéutico como farmacológico. Este conocimiento es determinado e incorporado por los usuarios, incrementado por las experiencias de vida personal, en donde se incorpora la información adquirida con el paso de los años o a través de experiencias de otros individuos. En el caso específico del tratamiento dietoterapéutico, gran parte de los entrevistados perciben que un bajo nivel de adherencia significará un alto riesgo de morbimortalidad a largo plazo.

La mayoría de los adultos mayores entrevistados conocen los beneficios que otorga la adherencia al tratamiento dietoterapéutico, el que se traduce, según sus respuestas, principalmente en el control de su patología. Los usuarios conocen el tipo de alimentación que deben seguir. En sus respuestas, indican, cantidad, frecuencia y tipos de alimentos permitidos en la dieta de una persona diabética.

Referente al tipo de dieta, destacan que se caracteriza por ser restrictiva y de alto costo para la población en estudio. Ambos puntos del tratamiento dietoterapéutico son percibidos como obstáculos para poder lograr una adherencia exitosa al tratamiento.

La percepción que tienen los individuos del estudio, respecto a los contenidos entregados por parte del nutricionista en sus controles sobre su tratamiento y la patología de base, es que estos últimos son entregados superficialmente, debido al poco tiempo dedicado a la educación durante los controles. Esto conlleva a que la información sea poco clara y no entendida por los usuarios. Además, la mayoría de los pacientes refieren presentar dudas en algunos puntos de su tratamiento, sin embargo, estas no son aclaradas por temor de preguntar al profesional y ser estigmatizados como limitados en sus conocimientos respecto a su patología.

\section{CONCLUSIÓN}

La interpretación de los resultados de la investigación realizada permite concluir que los adultos mayores diabéticos atendidos por nutricionistas en el centro de Atención Primaria de Salud de la comuna de Yumbel, poseen conocimientos acerca de la DM2, referido a las posibles complicaciones que esta patología puede presentar y los tratamientos que debe realizar. Sin embargo, estos conocimientos no determinan un alto nivel de adherencia a la dietoterapia, excepto en aquellos usuarios que presentan algún tipo de secuela de esta patología, en las que en un mayor nivel de adherencia a esta última. Estos resultados permiten interpretar que un mayor conocimiento de la patología no determina una mayor adherencia a la dietoterapia por parte de adultos mayores de una zona rural del país.

Los factores cognoscitivos no sólo incluyen los conocimientos que presentan los adultos mayores sobre su patología de base, también incorporan atribuciones, creencias o sus expectativas frente a esta última. Estos componentes no fueron incluidos en esta investigación.

Es necesario seguir líneas de investigación incorporando otros factores, como son los ambientales o emocionales, que permitirán enriquecer la interpretación de las percepciones que los pacientes presentan y que influyen en la adherencia a dietoterapia de la DM2.

\section{RESUMEN}

La Diabetes tipo 2 es una de las enfermedades crónicas de gran prevalencia en el país. Entre las estrategias para su tratamiento se encuentra la educación, como herramienta que presenta el equipo de salud para modificar conductas de riesgo en estas personas, entregando a los usuarios conocimientos y otras estrategias para su autocuidado. El objetivo del estudio fue interpretar los 
factores asociados a los conocimientos que influyen en la adherencia al tratamiento dietoterapéutico en adultos mayores con Diabetes tipo 2 controlados por nutricionista en la Atención Primaria de Salud de la comuna de Yumbel. A través de un estudio cualitativo de tipo fenomenológico, se realizó una entrevista semi-estructurada a 14 usuarios en condiciones estándar, obteniendo como resultado la presencia de conocimientos de su patología, referido a las consecuencias y los tratamientos a realizar. Sin embargo, estos conocimientos no determinan un alto nivel de adherencia a la dietoterapia, excepto en el caso de presentar algún tipo de secuela. Por lo tanto, se concluye que los conocimientos de la patología no favorecen una mayor adherencia a la dietoterapia por parte de adultos mayores de una zona rural del país.

Palabras claves: diabetes tipo 2, educación, adultos mayores, estudios cualitativos.

Dirigir la correspondencia a:

Sra.

Claudia Troncoso P.

Facultad de Medicina. Campus San Andrés.

Universidad Católica de la Santísima Concepción.

Alonso de Ribera 2850.

Concepción. Chile

Teléfonos: 041-2735427 - 041-2735406

E-mail: ctroncosop@ucsc.cl

Agradecimientos: A los profesionales de la atención primaria de salud de la comuna de Yumbel, quienes brindaron su colaboración para la realización de esta investigación, en especial a las nutricionista Gisella Moncada y Cecilia Hermosilla Toledo.

\section{BIBLIOGRAFÍA}

1. Fagalde M., Solar J., Guerrero M. et al. Factores de riesgo de enfermedades crónicas no transmisibles en funcionarios de una empresa de servicios financieros de la Región Metropolitana. Rev Méd Chile. 2005:133(8):919-928.

2. Ministerio de Salud Argentino. Costo de tratamientos para enfermedades agudas y crónicas. Política Nacional de Medicamentos. 2004. pp. 919-928.

3. Organización Mundial de la Salud. Prevención de la Enfermedades Crónicas. Una inversión vital. Panorama general. 2005. pp.1-3.

4. Carrasco E.; Perez F.; Angel B., et al. Prevalencia de diabetes tipo 2 y obesidad en dos poblaciones aborígenes de Chile en ambiente urbano. Rev méd Chil, 2004; 132, (10): 1189-1197.

5. Ramirez M. Estrategia mundial sobre régimen alimentario, actividad física y salud. INCAP. Instituto de Nutrición de Centro América y Panamá. 2004.

6. Arteaga C. Alimentación Saludable.Facultad de Medicina. Universidad de Chile. 2004.

7. Pena M.; Bacallao J. La obesidad y sus tendencias en la Región. Rev Panam Salud Pública 2001; 10(2) 45-78.

8. Ministerio de Salud de Chile. Objetivos Sanitarios para la década 2000 -2010. Boletín de vigilancia en salud pública de Chile.

9. Organización Panamericana de la Salud. La diabetes en las Américas. Boletín Epidemiológico. 2001. Vol. 22. No 2. Pág. 1 [online]. [citado noviembre 2008], Disponible en la World Wide Web: http://www.paho. org/spanish/sha/be_v22n2.pdf

10. Ministerio de Salud de Chile. Garantía Explícita en salud. Guía Clínica. 2005. Diabetes Mellitus tipo 1.

11. American Diabetes Association. Report of the Expert Committee on the Diagnosis and Classification of Diabetes Mellitus The Expert Committee on the Diagnosis and Classification of Diabetes Mellitus. Diabetes Care 200;26:S5-S20.

12. Organización Panamericana de la Salud. Estrategia regional y plan de acción para un enfoque integrado sobre la prevención y el control de las enfermedades crónicas, incluyendo el régimen alimentario, la actividad física y la salud. 47o Consejo Directivo. 58a Sesión del Comité Regional. Septiembre del 2006.

13. Ministerio de Salud de Chile. DEIS (Departamento de Estadísticas e Información en Salud) 2005. [online]. [citado julio 2008], Disponible en la World Wide Web: http://www.minsal.cl/

14. Selli L., Papaléo L., Meneghel S., Torneros J. Técnicas educacionales en el tratamiento de la diabetes. Cad Saúde Pública 2005;21(5): 1366-1372.

15. Cabrera C., González G, Vega M. et al. Impacto de la educación participativa en el índice de masa corporal y glicemia en individuos obesos con diabetes tipo 2. Cad Saúde Pública 2004;20(1)275-281.

16. Lyra R, Oliveira M, Lins D. et al. Prevention of type 2 diabetes mellitus. Arq Bras Endocrinol Metab 2006; 50(2)239-249.

17. Ferreira S., Almeida B., Siqueira A., et al. Interventions on the prevention of type 2 diabetes mellitus: is it feasible a population-based program in our country?. Arq Bras Endocrinol Metab 2005;49(4)479483.

18. Miyar L. Impacto de un programa de promoción de la salud aplicado por enfermería a pacientes diabéticos tipo 2 en la comunidad. Rev Latino-Am Enfermagem 2003;11(6) 713-719.

19. Lanes, R. Nuevas evidencias en el manejo de la dia- 
betes mellitus tipo 2. Medifam 2002;12(9)85-92.

20. Cabrera C. et al. Efectos de una intervención educativa sobre los niveles plasmáticos de LDLcolesterol en diabéticos tipo 2. Salud Pública Méx 2001;43(6)556-562.

21. Shils M., Olson R. Nutrición en salud y enfermedad. $9^{a}$ edición. México, ed. Mc Graw Hill. Vol. II, 2002, pp 1598.

22. Moreno E., Miles J. Adultos Mayores por Regiones, Comunas y Porcentajes. Documento de trabajo. Catastro de población adulto mayor. Servicio Nacional del Adulto Mayor. Instituto Nacional de Estadística. 2003.

23. Ruz, Araya, Atalah, Soto. Nutrición y Salud Departamento de Nutrición. Facultad de Medicina. Universidad de Chile. Santiago. Chile. Primera Edición 1996. pp. 299.

24. Dansinger M., Gleason J., Grffith J. et al. Comparison of the Atkins, Ornish, Weight Watchers, and Zone Diets for Weight Loss and Heart Disease Risk Reduction. J Am Med Assoc 2005;293:43-53.

25. Ministerio de Salud de Chile. Manejo del Paciente con Sobrepeso u Obesidad. Programa a de Salud Cardiovascular. 2002.

26. Pastén F. Elaboración de diagnósticos sociales con la utilización de técnicas cualitativas de investigación. Universidad de Valparaíso. Facultad de Derecho y Ciencias Sociales. Escuela de Servicio Social. Editorial Edelva. 2000. pp 18.

27. Pérez C. ¿Deben estar las técnicas de consenso incluidas entre las técnicas de investigación cualitativa?. Rev Española Salud Pública. 2000; 74 (4). 319-321.
28. Álvarez JL. Cómo hacer investigación cualitativa. Fundamentos y metodología. Editorial Paidós Educador. 2005. pp. 24.

29. Avilán JM. Investigación cualitativa en salud pública. Gaceta Méd Caracas 2005; 113(3).

30. Calderón J, Solis J, Castillo O. et al. Efecto de la educación en el control metabólico en pacientes con diabetes mellitus tipo 2 del Hospital Nacional Arzobispo Loayza. Rev Soc Peruana Med Int 2003;16(1). pp 17-25.

31. García R, Suárez R. Resultados de la estrategia cubana de educación en diabetes tras 25 años de experiencia. Rev Cubana Salud Pública 2007; 33(2).

32. Selli L, Papaléo L., Meneghel S., Torneros J. Técnicas educacionales en el tratamiento de la diabetes. Caderno Saúde Pública 2005;21(5) pp 1366-1372.

33. Otero L, Zanetti M. Ogrizio M. El conocimiento del paciente diabético sobre su enfermedad, antes y después de la implementación de un programa de educación en diabetes. Rev Latinoam Enfermería 2008; 16(2)231-237.

34. Silva T, Feldman Ch, Lima M. et al Diabetes mellitus and arterial hipertensión control with educational and therapeutic groups in outpatient follow-up in Basic health unit. Saúde e Sociedade 2006;15(3)180-189.

35. Tejada L, Pastor M, Gutiérrez S. Efectividad de un programa educativo en el control del enfermo con diabetes. Investigación y Educación en Enfermería 2006;24(2):48-53.

36. Rojas V., Sevilla D. Estudio de necesidades para la creación de un programa educativo para diabéticos. Médico Familia. 2004;12(1) 23-26. 